\title{
Comparative analysis of three mode-selection methods for solid-state lasers
}

\author{
Yan Sun ${ }^{*}$ \\ Air Force Aviation University, Changchun 130022, China
}

\begin{abstract}
Keywords: mode-selection, Iris diaphragm, telescope, gaussian mirror

Abstract. In the $\mathrm{Nd} 3+: \mathrm{YAG}$ pulsed Q-switched laser system, the selective output of several low order modes of TEM $\mathrm{TE}_{00}, \mathrm{TEM}_{01}$ and $\mathrm{TEM}_{11}$ is realized by adding a iris diaphragm, a telescope or a Gaussian mirror at an appropriate position in the resonator, and the three are compared and analyzed.
\end{abstract}

\section{Principle of transverse mode-selection for solid-state laser}

The advantages of laser are high power, good directivity, monochromaticity and coherence. In many cases,we hope that the laser can output a single mode. The laser output mode is divided into transverse mode and longitudinal mode.The factors affecting transverse and longitudinal modes are different, so the mode-selection method is different[1-2].The possible excitation mode of a fixed resonator is closely related to the cavity structure,the loss and the excitation level.Low order mode output is related to a small beam divergence Angle,so a certain transverse mode-selection technique must be adopted to obtain a single fundamental mode output [3].Suppose the reflectivity of the mirrors at both ends of the laser cavity are $\mathrm{r} 1$ and $\mathrm{r} 2$, the one-way loss is set to $\delta$, the one-way gain coefficient is $\mathrm{G}$, and the laser working material length is $\mathrm{L}$, then the initial light intensity of a certain transverse mode (TEMmn) is I0.After the light goes through a round trip in the resonant cavity, due to the influence of gain and loss, its light intensity becomes

$$
I=I_{0} r_{1} r_{2}(1-\delta)^{2} \exp (2 G L)
$$

The threshold condition is

$$
I \geq I_{0}
$$

It can be obtained

$$
r_{1} r_{2}(1-\delta)^{2} \exp (2 G L)>1
$$

Now we consider the two lowest-order transverse modes $\mathrm{TEM}_{00}$ and $\mathrm{TEM}_{01}$, their oneway losses are represented by $\delta_{00}$ and $\delta_{10}$, respectively. It is considered that the gain coefficient of the active medium for each transverse mode is the same. When the following two inequalities are satisfied at the same time:

\footnotetext{
*Corresponding author: 3507549885@qq.com
} 


$$
\begin{aligned}
& \sqrt{r_{1} r_{2}}\left(1-\delta_{00}\right) \exp (G L)>1 \\
& \sqrt{r_{1} r_{2}}\left(1-\delta_{10}\right) \exp (G L)<1
\end{aligned}
$$

At this time, the laser can realize fundamental mode $\left(\mathrm{TEM}_{00}\right)$ operation [4].

\section{Transverse mode-selection method}

An important parameter of the spherical mirror cavity is Fresnel number:

$$
N=\frac{a^{2}}{\lambda L}
$$

where, $a$ is the radius of the effective aperture in the cavity and $L$ is the cavity length. Diffraction loss is a function of Fresnel number $N$. In laser devices with low gain, the fundamental transverse mode can be selected by appropriate cavity parameter design.

The transverse mode-selection methods can be divided into two categories.One is to change the structure and parameters of the resonant cavity to obtain a larger difference in diffraction loss and improve the mode-selection performance of the resonant cavity. This method is called unstable cavity mode-selection. Gas lasers mostly adopt this method.The other is to insert additional mode-selection elements into a fixed resonant cavity to improve the mode-selection performance. In this paper, three methods of selecting mode by diaphragm, telescope and Gaussian mirror are studied.

The principle of diaphragm mode-selection is as follows. The light waist of the highorder mode is larger than that of the fundamental mode. If the aperture of the diaphragm is chosen properly, a part of the light beam of the high-order mode can be covered, and the fundamental mode can pass smoothly.According to the diffraction theory, the insertion of a small aperture in the cavity is equivalent to the reduction of the cross-sectional area of the cavity mirror, that is, the Fresnel number $\mathrm{N}$ of the cavity is reduced, so the diffraction loss of each mode is increased.As long as the diaphragm aperture is chosen properly, the fundamental mode can be selected. In practical work, it is usually according to the above theory to choose a small hole radius first, and then determine the size of the small diaphragm through experiments, or use the variable diaphragm to select the appropriate small hole according to specific requirements.Although the mode-selection for the aperture diaphragm is simple in structure and convenient in adjustment, due to the limitation of the aperture, the volume of the fundamental mode in the cavity is small, and the volume of the working material cannot be fully utilized. The output laser power is relatively small. When the power density in the cavity is high, the diaphragm is easy to be damaged .

The principle of telescope mode-selection is as follows.Suppose the magnification of the telescope is M. Due to the beam expansion effect of the telescope, the mode volume can be enlarged by $\mathrm{M}^{2}$ times when the beam passes through the laser working material.Because the focal length of the telescope is very short, it has a strong focusing or divergence effect on the beam, which causes the divergence Angle of each mode to expand by M times. The fundamental mode has the smallest divergence angle. If the size of the diaphragm is chosen to be similar to the spot of the fundamental mode, the higher order mode will be blocked by the diaphragm and lose, and only the fundamental mode can be retained.

Gaussian lens mode-selection is a kind of special cavity lens selection. The reflectivity of the cavity mirror presents a Gaussian distribution. The selectivity of the endoscope provides feedback to the fundamental mode, but the high order mode loss is large, thus the fundamental mode oscillation is realized.This technique can effectively select the mode 
output, realize the large mode volume operation and improve the single mode output power of the laser.

\section{Experimental study on mold selection}

\subsection{Experimental device}

The experimental device is shown in Figure 1.

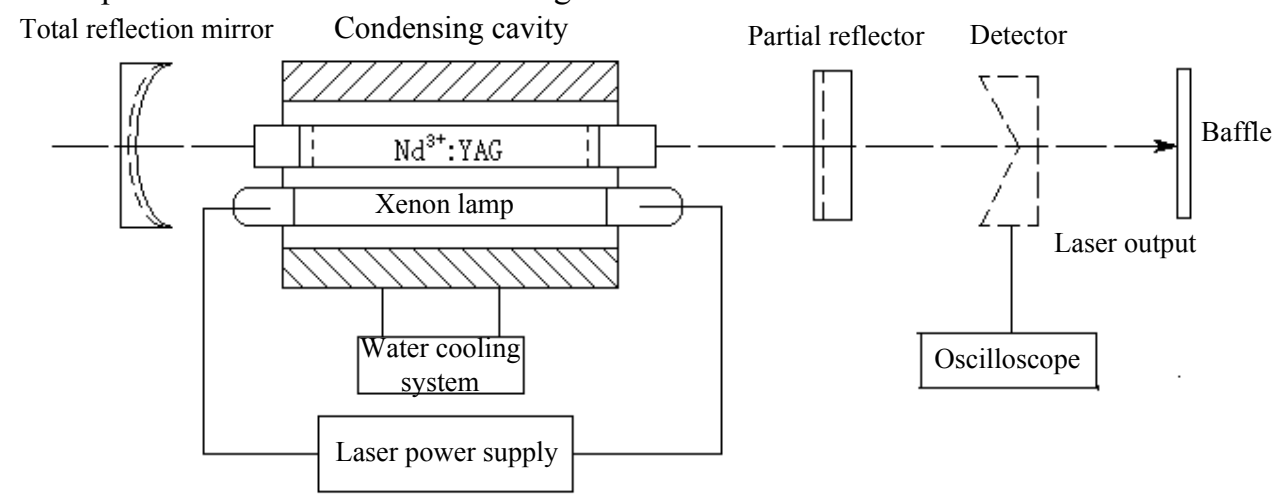

Fig. 1. Nd3+: YAG laser device diagram.

The performance indicators of the laser are as follows (600V):

Laser crystal shape: cylindrical shape Nd3+:YAG;Working wavelength: 1064nm;Frequency doubling wavelength (extracavity frequency doubling): 532nm;Single pulse energy: 400mJ; Repetition frequency: 1/3/10Hz (single manual) ; Pulse width: $<100 \mu \mathrm{s}$; Beam diameter: $6 \mathrm{~mm}$; Height of beam center from platform: $105 \mathrm{~mm}$; Conversion efficiency: $2.24 \%$; Power supply: $220 \mathrm{~V} / 50 \mathrm{~Hz}$.

\subsection{Mode-selection experiment}

\subsubsection{Diaphragm mode-selection}

This experiment adopts the iris diaphragm with the size of $\Phi 1.5 \mathrm{~mm}, \Phi 2 \mathrm{~mm}, \Phi 2.5 \mathrm{~mm}$. The experiment is carried out by changing the size of the diaphragm and the length of the resonator cavity at the state of output static laser.

A iris diaphragm is added to the apparatus in the experiment. The optical path has to be readjusted because of the deviation caused by the addition of new components. When adjusting the diaphragm, it needs to be carefully adjusted up, down, left, and right, as well as the pitch angle, so that the collimated light completely passes through the diaphragm. Finally, make the centers of the elements coaxial and make the beams parallel.

The Light spot aperture is $\Phi 1.5 \mathrm{~mm}, \mathrm{~L} 2$ is $200 \mathrm{~mm}$, the cavity length of the resonator is $482 \mathrm{~mm}(\mathrm{~L} 1=100 \mathrm{~mm}, \mathrm{n} 0 \mathrm{~d}=1.82 \times 100 \mathrm{~mm}=182 \mathrm{~mm})$, and the laser output spot is shown in Figure 2.It can be seen from the figure that the laser output mode is TEM00 mode at this time.Figure 3 shows the pulse waveform detected by the oscilloscope. 


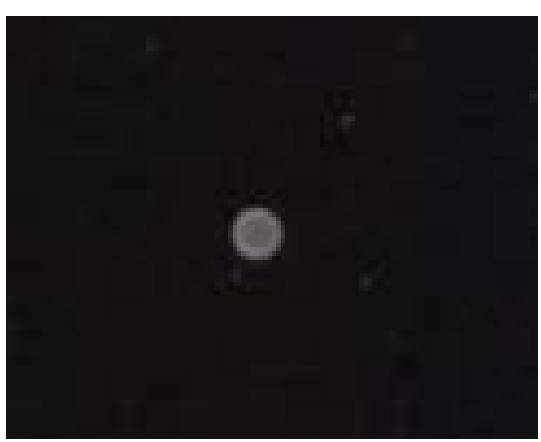

Fig. 2. $\mathrm{TEM}_{00}$ spot.

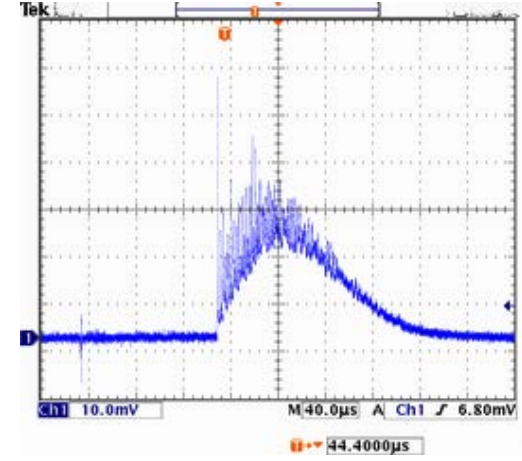

Fig. 3. $\mathrm{TEM}_{00}$ waveform.

Further we change the diaphragm aperture and L2, and repeat the above experimental steps.When the diaphragm aperture is $\Phi 2 \mathrm{~mm}$ and the cavity length is $482 \mathrm{~mm}$ (the position of the diaphragm and cavity length remain unchanged), the output spot is shown in Figure 4, and the mode is $\mathrm{TEM}_{01}$. The output waveform is shown in Figure 5. The spot aperture measured at this time is $\Phi 0.9 \mathrm{~mm}$.

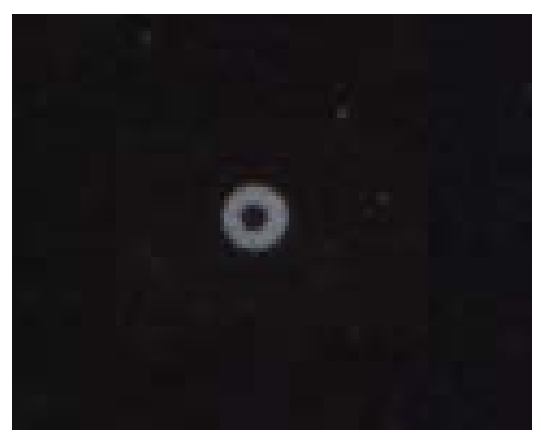

Fig. 4. $\mathrm{TEM}_{01}$ spot.

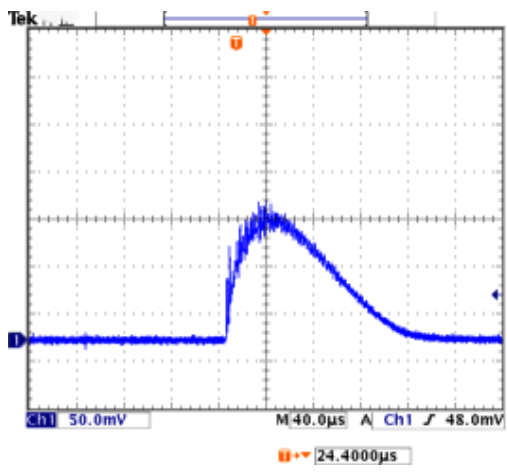

Fig. 5. TEM $\mathrm{T}_{01}$ waveform.

We change the diaphragm aperture again, keep the length of the resonator cavity unchanged, repeat the above experimental steps to obtain the output of the TEM11 mode. The output spot is shown in Figure 6, the output waveform is shown in Figure 7 and the measured spot aperture at this time is $\Phi 0.65 \mathrm{~mm}$.

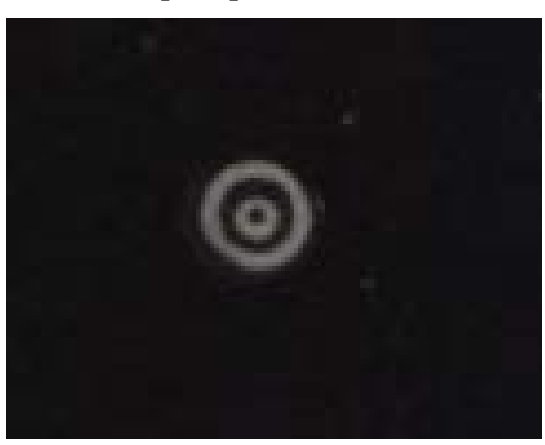

Fig. 6. $\mathrm{TEM}_{11}$ spot.

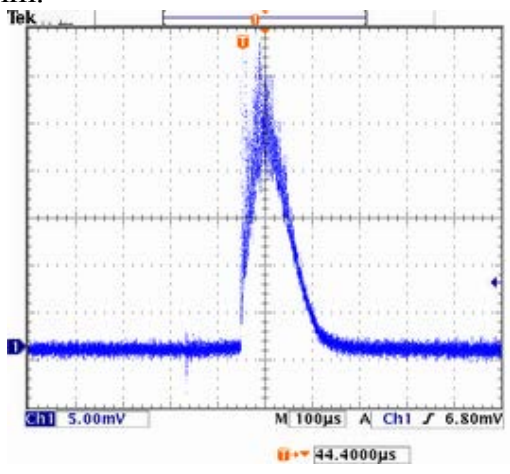

Fig. 7. $\mathrm{TEM}_{11}$ waveform. 
According to the theory of diaphragm mode-selection, the pulsed solid state laser is used to suppress the higher order modes, and the low order modes of TEM00, TEM01 and TEM11 are selected.

In the experiment, the diaphragm aperture used in the fundamental mode-selection is $\Phi 1.5 \mathrm{~mm}$, and the cavity length is $482 \mathrm{~mm}$, which is basically consistent with the theoretically calculated the diaphragm aperture when the fundamental mode is output from $1.54 \mathrm{~mm}$ to $1.87 \mathrm{~mm}$, and the resonant cavity length is $500 \mathrm{~mm}$. The diaphragm modeselection theory is introduced. Insert a diaphragm in the device, and when the laser is static output, the output waveform amplitude and output energy measurement values are shown in Table 1 under different xenon lamp voltages.

Table 1. Static energy with diaphragm mode-selection (threshold value of 420V).

\begin{tabular}{ccc}
\hline Xenon lamp voltage $(\mathrm{V})$ & Waveform amplitude $(\mathrm{mV})$ & Output energy $(\mathrm{mJ})$ \\
\hline 560 & 56.32 & 17.93 \\
600 & 79.43 & 25.30 \\
640 & 98.57 & 31.39 \\
680 & 132.3 & 42.13 \\
720 & 161.7 & 51.50 \\
\hline
\end{tabular}

\subsubsection{Telescope mode-selection}

The magnification of the telescope used in the experiment is 3, the total reflection mirror diameter is $\Phi 25 \mathrm{~mm}$, the curvature radius is $4 \mathrm{~m}$, and it has high reflectivity for $1.064 \mu \mathrm{m}$ wavelength. The output mirror size is $\Phi 25 \mathrm{~mm}$, and the reflectivity for $1.064 \mu \mathrm{m}$ wavelength is $10 \%$. Before the experiment, it must be finely adjusted to make the centers of the components coaxial.

The laser output pulse width at this point is shown in figure 8 by an oscilloscope .The measured spot diameter is $\Phi 3.25 \mathrm{~mm}$.

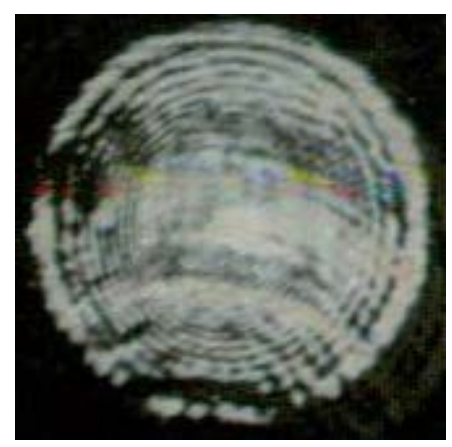

Fig. 8. $\mathrm{TEM}_{00}$ spot.

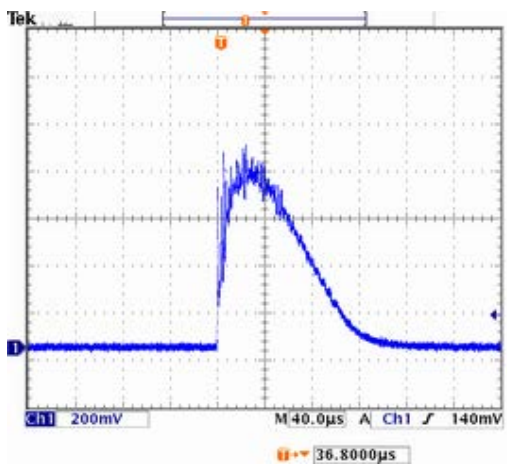

Fig. 9. TEM $\mathrm{T}_{00}$ waveform.

Table 2. Static energy with telescope mode-selection (threshold of 410V).

\begin{tabular}{ccc}
\hline Xenon lamp voltage $(\mathrm{V})$ & Waveform amplitude $(\mathrm{mV})$ & Output energy $(\mathrm{mJ})$ \\
\hline 560 & 35.03 & 11.57 \\
600 & 146.8 & 46.75 \\
640 & 252.0 & 80.25 \\
680 & 390.0 & 124.2 \\
720 & 536.0 & 170.7 \\
\hline
\end{tabular}

When the static laser output is inserted into the device, the measured values of output waveform amplitude and output energy under different xenon lamp voltages are shown in Table 2. 


\subsubsection{Gaussian mirror mode-selection}

We replace part of the reflector in the experimental device with a Gaussian mirror.As the Gaussian mirror is a convex lens, in the process of installation and adjustment, the collimating head should pass through the deepest point of the coating film, and then adjust the collimation of its optical path.Only when the central coaxial adjustment of each element is good, a better spot output can be obtained.The laser output pulse width at this time is detected by an oscilloscope,as shown in Figure 11.

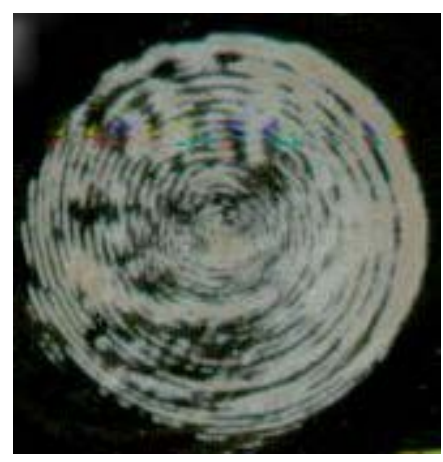

Fig. 10. TEM $\mathrm{T}_{00}$ spot.

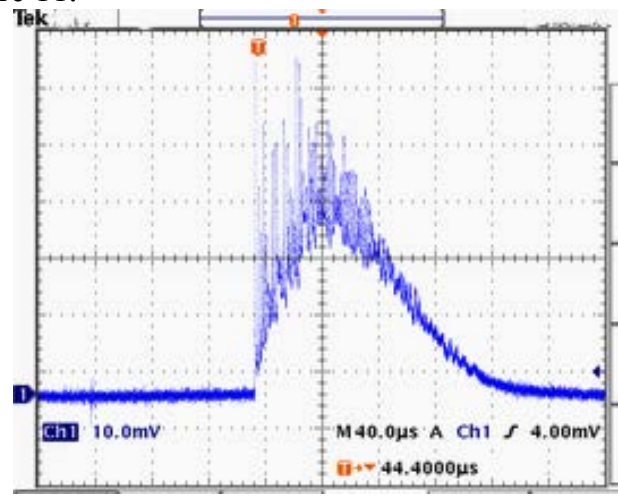

Fig. 11. $\mathrm{TEM}_{00}$ waveform.

The partial reflector is replaced with a Gaussian mirror. When the laser is output statically, the output waveform amplitude and output energy measurement values are shown in Table 3 under different xenon lamp voltages.

Table 3. Static energy with Gaussian mirror mode-selection (threshold of 400v).

\begin{tabular}{ccc}
\hline Xenon lamp voltage $(\mathrm{V})$ & Waveform amplitude $(\mathrm{mV})$ & Output energy $(\mathrm{mJ})$ \\
\hline 560 & 47.66 & 15.18 \\
600 & 118.09 & 37.61 \\
640 & 219.20 & 69.81 \\
680 & 363.55 & 115.78 \\
720 & 499.26 & 159.00 \\
\hline
\end{tabular}

\subsection{Data analysis}

According to the above data, draw the relationship curve between the output energy of the system and the voltage of the xenon lamp when the diaphragm, the telescope and the Gaussian mirror are inserted, as shown in Figure 12.

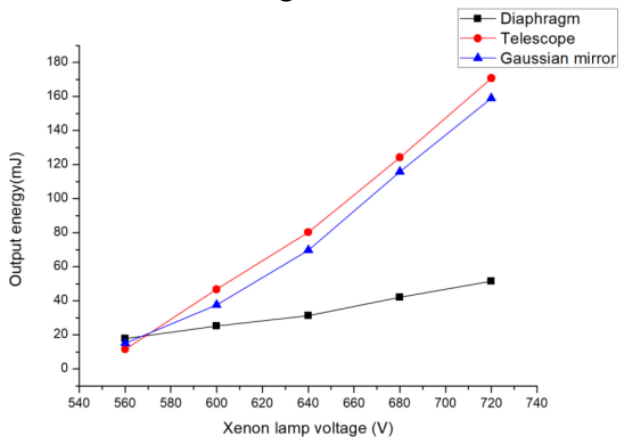

Fig. 12. Relationship between laser output energy and pump voltage. 
It can be seen from the figure that under the same voltage, the output energy of the three increases with the voltage, and the output energy of the system inserted into the telescope increases the fastest. Because the telescope and Gaussian mirror selection can obtain a larger mode volume, the output pulse energy is 2-3 times larger than that of the diaphragm mode-selection.

The diaphragm mode-selection is relatively simple in structure, easy to adjust, and the device development cost is low.However, due to the diaphragm limitation, the basic mode beam in the cavity is thin and the mode volume is small, so the laser output energy obtained is low. The insertion of the telescope in the cavity is not only beneficial to the operation of the fundamental mode with large volume, but also can be used to compensate the thermal lens effect caused by the heavy frequency operation of the device to some extent.At this point, the telescope performs two different functions: first, it reduces the size of the output beam and increases the diffraction loss of the higher order mode; On the input side, the telescope amplifies the beam to match the diameter of the rod.Second, a telescope is a focal length variable, so it can be adjusted no matter where it is in the resonator.However, compared with the diaphragm, its structure is complex and the telescope itself has a certain amount of light energy loss, so the development cost of this method is high. The unsteady cavity has high efficiency, low sensitivity to disturbance and compact structure.

\section{Summary}

In this paper, three methods are used to realize the mode-selection of solid state laser and improve the transverse mode output of laser. The three methods are analyzed from the aspects of technical difficulty and influence on laser energy output, and the advantages and disadvantages of the three methods are compared, which can provide reference for basic design of solid state laser.

\section{References}

1. Zhang Xue, Ge Wenqi, Yu Jin, etc. Etalon accuracy and mode-selection performance in pulsed single longitudinal mode lasers. Power Laser and Particle Number, 2017, Vol.29:21-27.

2. Wan Hongdan, Ding Zuoqin, Wang Jie, etc. Single longitudinal mode narrowband fiber laser based on high roundness microsphere cavity mode-selection.China Laser. 2017, Vol. 44:30-35.

3. Lu Baida. Laser Optics. Higher Education Press. 2003.361.

4. Liu Jinghai. Xu Rongpu. Laser Devices and Technology. Beijing: University of Science and Technology Press, 2004.111. 